\title{
Learning to live together: mutualism between self-splicing introns and their hosts
}

\author{
David R Edgell ${ }^{1 *}$, Venkata R Chalamcharla ${ }^{2,3}$ and Marlene Belfort2*
}

\begin{abstract}
Group I and II introns can be considered as molecular parasites that interrupt protein-coding and structural RNA genes in all domains of life. They function as selfsplicing ribozymes and thereby limit the phenotypic costs associated with disruption of a host gene while they act as mobile DNA elements to promote their spread within and between genomes. Once considered purely selfish DNA elements, they now seem, in the light of recent work on the molecular mechanisms regulating bacterial and phage group I and II intron dynamics, to show evidence of co-evolution with their hosts. These previously underappreciated relationships serve the co-evolving entities particularly well in times of environmental stress.
\end{abstract}

One of the most intricate relationships in biology is that between a host and a parasite. Almost all organisms studied so far harbor mobile genetic elements and/or their derivatives. At the genomic level, the traditional view of mobile elements is that they provide seemingly little or no benefit to the host while parasitizing the host's cellular machinery to promote element mobility through complex molecular pathways [1,2]. The host's response to these elements is primarily defensive, as evidenced by the many forms of negative regulation that downregulate the activity of mobile elements [3-8]. The persistence of a mobile element in a given population is thus the result of a delicate balance between an excessive mutational burden on the host caused by the element's unrestricted activity, and excessive negative regulation imposed by the host on the element to limit mobility. While the relationship between host and mobile element is often viewed as a molecular arms race [9], recent experimental

\footnotetext{
*Correspondence: dedgell@uwo.ca, belfort@wadsworth.org

'Department of Biochemistry, Schulich School of Medicine and Dentistry,

The University of Western Ontario, London, Ontario, Canada N6A 5C1

Full list of author information is available at the end of the article
}

data argue that the relationship is more elaborate than previously appreciated.

\section{Mobile introns: ribozymes with baggage}

One group of mobile genetic elements comprises the group I and II introns. These sequences interrupt protein-coding and structural RNA genes in all domains of life and can be considered as molecular parasites. When the gene is transcribed into RNA, the intron sequence acts as a ribozyme (an RNA with enzymatic activity), which removes the intron sequence from the primary RNA transcript, thus limiting the phenotypic cost associated with insertion of the element into a host gene and promoting their maintenance in the genome. In the case of group I and II introns, the host-parasite relationship is enriched by the fact that the introns themselves have been invaded by smaller parasitic elements - genes that encode mobility-promoting activities that enable the DNA element to move within and between genomes [10]. Thus, at least two levels of parasitism exist for mobile introns: the intron in the host gene it interrupts, and the invading gene in the intron. Collectively, the intron and its encoded mobility protein (often termed an intronencoded protein, IEP) collaborate to form a composite mobile element that utilizes host DNA replication, recombination and repair pathways to spread [11], while the ribozyme activity ensures that it does not disrupt the function of genes into which it is inserted. Accordingly, it has become evident that there is an extraordinary degree of co-evolution among IEPs, the introns that house them, and the host organism. This review highlights several recent studies probing the interplay among self-splicing introns in bacterial and phage genomes, their genes, and their bacterial and phage hosts.

\section{Group I introns}

Group I introns commonly inhabit bacterial, organellar, bacteriophage and viral genomes, and the ribosomal RNA genes (rDNA) of eukaryotes, and produce a selfsplicing RNA [12]. Group II introns have a similar distribution, except that they are not found in eukaryotic nuclear genes. Group I and group II introns show little primary sequence conservation, yet their RNAs each 
adopt characteristic secondary and tertiary structures necessary for ribozyme activity [13,14] (Figure 1a,b). Moreover, the introns can tolerate the insertion of large amounts of sequence into terminal loops of the ribozyme secondary structure with little or no effect on splicing, providing convenient 'hiding' spots for parasitic genes.

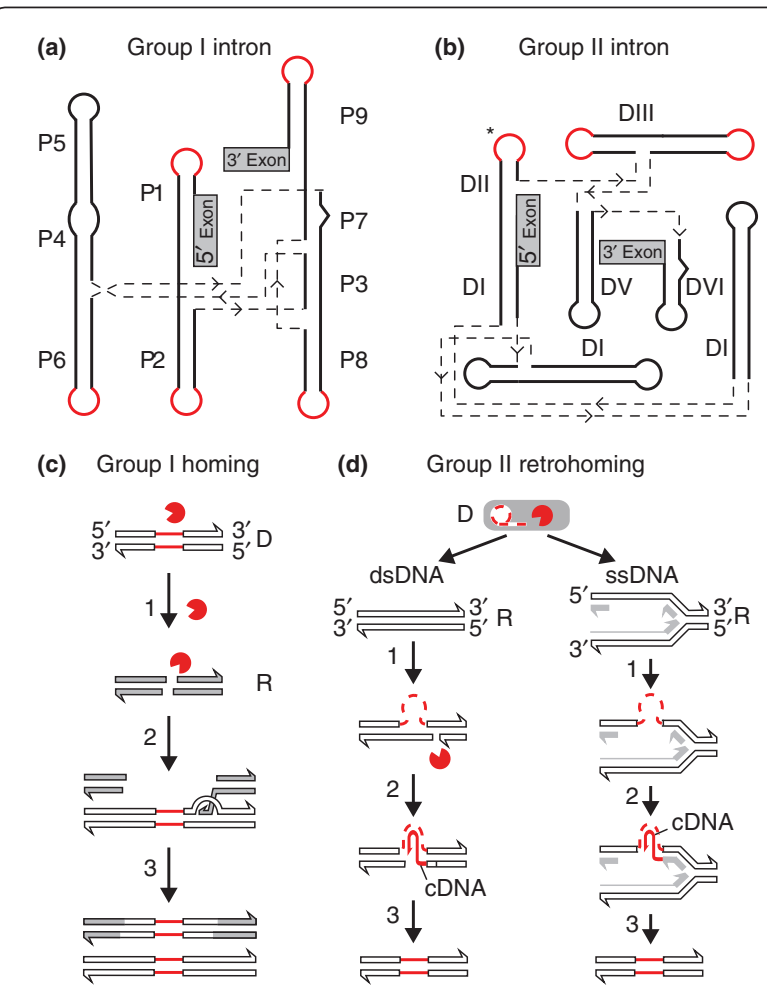

Figure 1. Models of group I and group II introns and their 'homing' mechanisms. (a,b) Schematic representations of (a) group I and (b) group II intron secondary structures [13,37]. In both cases, secondary structures are represented by solid lines indicating conserved stem-loop structures, named P1 to P10 for group I introns, and DI to DVI for group II introns. The positions of ORFs and other insertions are depicted by solid red lines. The asterisk $(*)$ next to domain II of group II introns indicates bioinformatic predictions of the ORF start sites, but these remain uncharacterized. Dashed gray lines indicate joining regions of unpaired nucelotides, with arrows indicating a $5^{\prime}-3^{\prime}$ orientation. The $5^{\prime}$ and $3^{\prime}$ exons are indicated by grey rectangles. (c) Homing of a group I intron. In this DNA-based mobility pathway, the intron donor (D) expresses the intron endonuclease (red enzyme symbol) (step 1). After cleavage of the allelic intron recipient sequence (R) at the homing site (step 2) the donor and recipient engage in double-strand break (DSB) repair to generate two introncontaining alleles. (d) Group II intron retrohoming by means of an RNA intermediate. The intron donor (D) in this case is the spliced intron lariat RNA (dashed red line), whereas the recipient (R) can be either double-stranded DNA (dsDNA) or single-stranded DNA (ssDNA), as at a replication fork. A ribonucleoprotein complex between the RNA and the IEP catalyzes a reverse splicing (step 1). In the dsDNA pathway the IEP then cleaves the second strand to generate the primer for cDNA synthesis by the IEP, whereas in the SsDNA pathway an Okazaki fragment at the replication fork (solid gray line) acts as a primer (step 2). Second-strand cDNA synthesis followed by repair completes the retromobility reactions (step 3).
Many group I introns move by a DNA-based transposition mechanism known as 'homing. Such introns harbor genes encoding so-called homing endonucleases, site-specific but sequence-tolerant DNA endonucleases that introduce double-strand breaks (DSBs) in cognate alleles that lack the intron, initiating intron mobility via a DSB-repair process [11] (Figure 1c). The outcome of a homing event is the unidirectional movement of the intron and endonuclease open reading frame (ORF) to an unoccupied allele, leaving a copy of the intron in its original location (Figure 1c). Group I introns can also harbor other 'baggage'. Many group I introns in organellar genomes encode maturases proteins that help promote intron splicing by a variety of mechanisms $[15,16]$. Some maturases also function in trans to promote splicing of other group I introns in the same genome $[17,18]$. Interestingly, many maturases characterized so far are degenerate or bifunctional homing endonucleases of the LAGLIDADG class - so named for their conserved sequence motif - that have acquired an RNA chaperone activity independent of their DNA endonuclease activity $[19,20]$. Group I introns can also harbor ORFs unrelated to mobility or splicing $[21,22]$, as exemplified by the astonishing case of an approximately 18-kilobase-long intron inserted in the mitochondrial ND5 gene of the mushroom coral Discosoma that encodes 15 mitochondrial genes in the P8 loop of the intron [23,24]. Interestingly, these 15 genes include both the small and large subunit rRNA genes and the cox1 gene, which is interrupted by another selfsplicing group I intron.

Some bacterial group I introns have been invaded by mobile elements other than those that encode homing endonucleases. Notable among these are the chimeric intron/insertion sequence (IS) elements (IStrons) of Clostridium that contain an IS605-like element inserted at the 3' end of the intron [25]. It is not known, however, whether the chimeric intron/IS element is mobilized by the IS605 machinery. Intriguingly, another unusual clostridial group I intron arrangement was recently found by a bioinformatic search for riboswitches [26], RNA structural elements that control gene expression through alternative secondary structures in response to binding of secondary metabolites. In this case, the tandem riboswitch/intron lies in the upstream region of a putative virulence factor gene, and sensing of cyclic diguanosyl-5'-monophosphate by the riboswitch controls choice of the 3' splice junction by the intron to modulate expression of the virulence factor.

While many ORFs embedded within group I introns are entirely located in loop regions, a surprising number of ORFs extend beyond peripheral loops to contribute nucleotides to more distant regions of the intron that form key structural elements needed for splicing [27]. The 


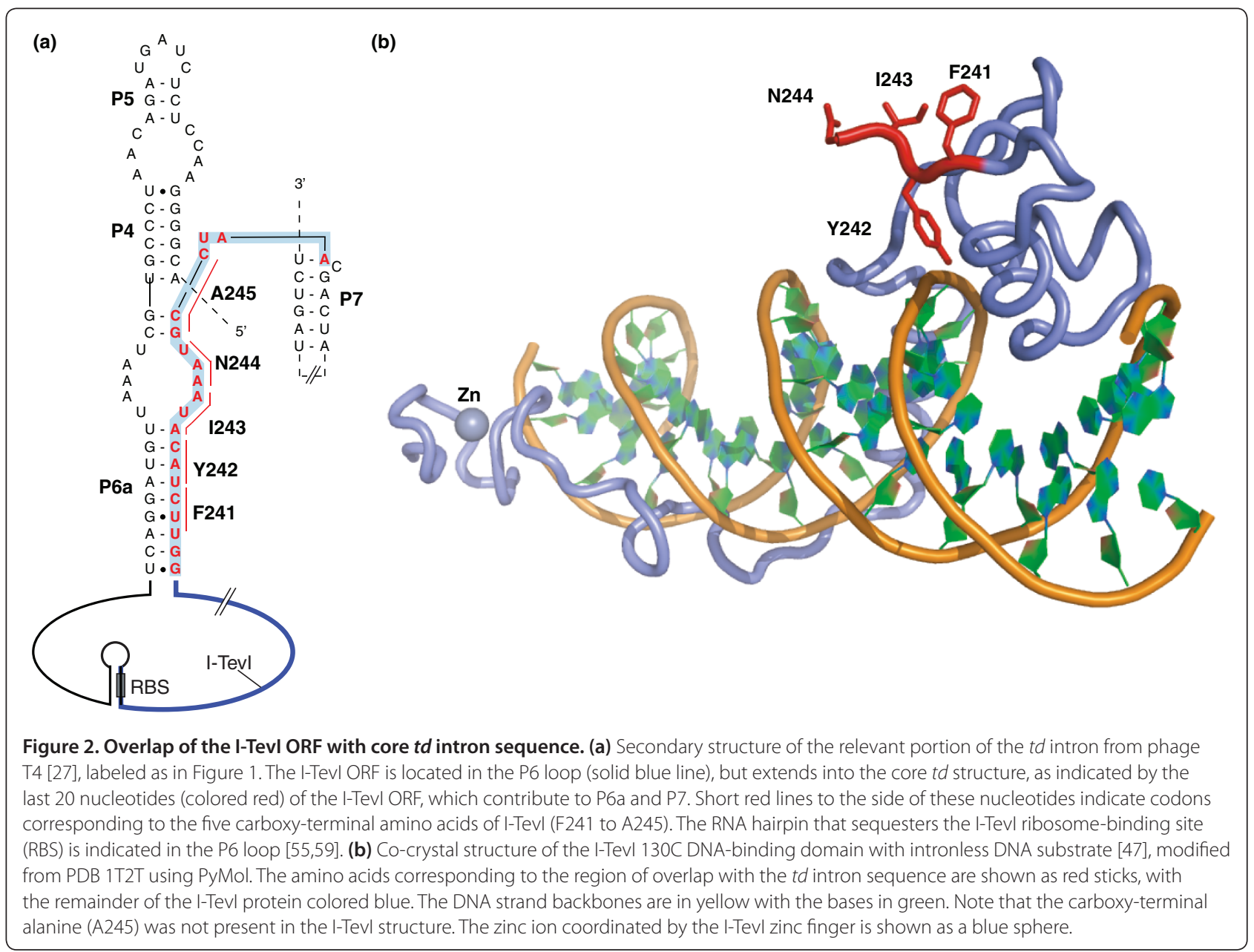

extent of the contribution of ORF sequence to ribozyme structural elements varies depending on the particular intron-ORF arrangement. For instance, in the wellstudied bacteriophage T4t $t d$ intron, the 3 ' end of an ORF called I-TevI (which encodes a homing endonuclease of the GIY-YIG type, again named for a conserved sequence motif) contributes 20 nucleotides that form part of the P6a, P6.0 and P7 structures that are essential for splicing of the intron [28] (Figure 2a). In other cases, the extent of the overlap is greater, involving the $5^{\prime}$ end as well as the $3^{\prime}$ end of the endonuclease ORF (Table 1). It should be noted, however, that the extent of overlap noted is based on predictions of endonuclease ORFs, and it is possible that many cases of extensive overlap result from incorrect bioinformatic identification of the 5' and 3' ends of endonuclease genes. Regardless of this, the presence of an endonuclease ORF within a highly structured RNA molecule poses a number of fascinating evolutionary and functional questions. Specifically, how did composite mobile introns evolve, and what are the functional consequences of translation of endonuclease ORFs from within such highly structured RNA molecules?

Table 1 Examples of ORF overlap with core group I intron sequences

\begin{tabular}{lccccc}
\hline Organism & $\begin{array}{c}\text { Host gene } \\
\text { interrupted }\end{array}$ & $\begin{array}{c}\text { Endonuclease } \\
\text { family }\end{array}$ & $\begin{array}{c}\text { Insertion site } \\
\text { within intron }\end{array}$ & $\begin{array}{c}\text { Overlap with } \\
\text { intron (nucleotides) }\end{array}$ & $\begin{array}{c}\text { Structural } \\
\text { element overlap }\end{array}$ \\
\hline Bacillus thuringiensis sup. pakistani [90] & nrdF & GIY-YIG & P6a & 56 & P6a/P7/P7.1/7.1a \\
Bacillus phage SPO1 [91] & DNA polymerase & HNH & P8 & 9 & P8 \\
Synechococcus lividus [92] & rDNA LSU & LAGLIDADG & P8 & 81 & P6/P7/P3/P8/P9.0/P9 \\
Synechocystis PCC 6803 [93] & tRNAfmet & PD-(D/E)-XK & P1 & 61 & P1/P2 \\
Physarum polycephalum [94] & rDNA LSU (nucleus) & His-Cys box & P1 & 0 & None \\
\hline
\end{tabular}




\section{Group II introns}

Similar questions can be asked regarding group II introns, which are found in similar niches to group I introns, but not in nuclear genomes [14]. Group II introns all have a common ribozyme structure consisting of six helical domains (Figure 1b) [29,30]. Their mobilitypromoting IEPs are typically encoded within domain IV, and the introns move via an RNA-based mechanism known as 'retrohoming. Unlike their group I intron counterparts, the group II IEPs are multifunctional proteins containing maturase $(\mathrm{X})$, reverse transcriptase (RT) and DNA-binding (D) functions in addition to DNA endonuclease (En) activity. The maturase activity facilitates intron splicing by stabilizing the catalytically active RNA conformation, while the RT, D and En functions aid in RNA-based mobility pathways. In this type of movement, the spliced intron lariat RNA invades either double- or single-stranded DNA ((Figure 1d). As well as retrohoming to allelic target sites, group II introns can transpose to non-allelic sites $[11,14]$.

Group II introns can also be invaded by elements encoding proteins other than the multifunctional IEPs. These elements include, but are not limited to, simple LAGLIDADG endonuclease ORF insertions in domain III $[31,32]$. Another arrangement produces the so-called twintrons, in which a group II intron has inserted into another group II intron, as in the case of the $p s b$ locus in Euglena gracilis chloroplast DNA and the Tell introns in the cyanobacterium Thermosynechococcus elongatus [33-35]. Whereas some insertions functionally 'split' the group II intron and interfere with intron splicing, others, such as some eukaryotic organellar introns, allow trans-splicing [36].

Recent crystallographic studies on the ribozyme structure of the Oceanobacillus iheyensis group II intron Oi5g revealed that coaxial stacking of domain IV with its neighboring domain III projects domain IV away from the ribozyme core, probably preventing nonproductive interactions of the IEP coding sequence with the ribozyme core [37]. Likewise, the positioning of domains II and III away from the ribozyme suggests that they can accommodate additional sequence [29,38]. Although domains II, III and IV may enhance splicing efficiency, they are not strictly required for catalysis, making them hospitable sites for invasive elements. In bacteria, IEPs are encoded entirely within loops of their host group II introns, and possess regulatory features such as promoters and ribosome-binding sites that are distinct from those that control expression of the host gene in which the intron resides [39]. In contrast, in organellar genomes, ORFs embedded within group II introns are regulated by promoters in the upstream exons $[40,41]$. Thus, the intron ORFs are initially translated as fusion proteins with the 5 ' exon and require subsequent proteolytic processing $[40,41]$.

\section{Visitors make themselves at home: core creep}

Many lines of evidence suggest that both group I and group II introns were ancestrally ORF-less, only to be invaded multiple independent times to create composite mobile elements. Notably, ORFs are located at different positions within introns; similar introns contain different ORFs; and similar ORFs occur in divergent introns. Several hypotheses have been put forward to explain the origin and evolution of mobile introns [42-46], with each hypothesis relying on illegitimate recombination pathways to create a composite mobile intron consisting of intron and endonuclease ORF. These hypotheses do not, however, address the evolutionary and functional ramifications of the overlap of protein ORFs with key structural elements of their host introns. Also worth considering are the multiple selective pressures on ORFs that extend into the ribozyme core: the ORF sequence must evolve in such a way so as not to accumulate substitutions that adversely affect endonuclease activity (and hence affect the spread and retention of the intron in populations); while at the same time it must co-evolve with disparate regions of the intron to ensure that secondary structure elements necessary for splicing are maintained by compensatory base-pairing interactions.

We propose an alternative scenario for invasion of introns by ORFs in which ORF insertion into peripheral loops of the introns was favored, such that the ORF sequence did not overlap with core intron sequences, thus limiting any phenotypic cost associated with reduced intron splicing. This scenario also avoids the requirement that the invading ORF would have to contain exactly the same nucleotides as it was replacing in order to maintain the crucial base pairing required for intron folding. Instead, we argue that the current overlap of intron ORFs with core intron sequences occurred after invasion by a process we term 'core creep'. Essentially, this is an extension of the coding region by mutation of an existing termination codon into one specifying an amino acid, so that the ORF is extended until the next occurrence of an in-frame termination codon. For intronencoded ORFs that underwent core creep, the next termination codon could lie within ribozyme core sequences, resulting in the overlap exhibited in many intron-ORF arrangements. Similarly, selection of an alternative initiation codon can account for the observation that the 5 ' ends of some endonuclease ORFs include intron core sequences.

Importantly, this hypothesis gives rise to a number of testable predictions. First, the length of the $5^{\prime}$ or $3^{\prime}$ extension should be variable for each independent case of endonuclease invasion, and the position of the initiation or termination codon should be influenced by the GC content of the intron because termination and initiation codons are slightly more AT rich than GC rich and the 
GC content of the intron will therefore influence the probability of mutation of a sense codon into a nonsense (stop) codon. The second prediction is that the sequence at the $5^{\prime}$ or $3^{\prime}$ ends of an endonuclease ORF that extends into the intron may not be essential for endonuclease function. In the case of I-TevI, the 20 nucleotides that extend into the $t d$ intron encode 6 amino acids on the carboxy-terminal end of I-TevI (out of a total 245 amino acids). In the crystal structure of the I-TevI DNA-binding domain bound to DNA representing either its homing target site or its target operator site, only one of the carboxy-terminal residues (Tyr242) makes a hydrogen bond to the phosphate backbone of the DNA substrate, clearly not providing any specificity to the interaction of I-TevI with its DNA substrate [47]. Bioinformatic searches with the I-TevI amino acid sequence also show that the carboxyl terminus is variable in length and composition (DRE, unpublished observations), implying that it is not critical for function.

\section{Don't bite the hand that feeds you: translational regulation of intron ORFs}

The successful spread and retention of mobile introns depends on expression of the mobility-promoting protein from within the intron, and on accurate splicing-out of the introns from the flanking exon sequences. For most group I introns, mobility and splicing are independent of each other, whereas for group II introns, and some organellar group I introns, these processes are not mutually exclusive. In these cases, translation of the IEP from the pre-splicing intron transcript is necessary for splicing because the IEP acts as an RNA maturase, in addition to facilitating mobility (reviewed in [14]). Furthermore, for group II introns, the spliced-out ribozyme is the agent of mobility, integrating into the DNA target [48] (Figure 1d). Thus, translation of intronencoded proteins must be carefully orchestrated so as not to interfere with intron-splicing pathways, and recent studies have revealed that diverse mechanisms are employed to regulate ORF expression and intron splicing.

One potential barrier to efficient intron splicing in bacterial and organellar genomes is the coupled nature of transcription and translation, which raises the possibility that ribosomes translating the RNA transcript could encounter the 5' exon-intron junction before the 3' splice site of the intron is transcribed, thus preventing the folding of critical intron structures and recognition of the correct splice sites by the intron. Ironically, a number of studies with bacterial group I introns have shown that translation of the exon upstream of the 5 ' splice site is necessary for efficient splicing, probably because a ribosome at this position acts as a 'chaperone' to prevent nonproductive interactions between exon and intron sequences that would disrupt the intron-folding pathway
[49-51]. Most group I introns also have an in-frame stop codon positioned immediately downstream of the 5 , exon-intron junction to prevent ribosome entry into the structured intron RNA. Ribosome entry into the intron core could also occur as a result of translation events that initiate at ORFs embedded within the intron. The various approaches to downregulating the translation of intronencoded ORFs in prokaryotic genomes include the presence of non-AUG initiation codons and nonconsensus ribosome-binding sites [52,53].

More complicated types of regulation are implied by numerous examples of ribosome-binding sites in introns that are sequestered by RNA secondary structure [5458]. Mutational analysis of one such RNA secondary structure that regulates translation of the I-TevI homing endonuclease revealed a pronounced splicing defect resulting from ribosome occupancy of intron sequences that form the crucial structures necessary for splicing [59]. A different strategy of regulating translation from within a bacterial group II intron has been revealed by detailed biochemical studies of the LtrA protein encoded within the LI.LtrB group II intron of Lactococcus lactis $[60,61]$. LtrA binds with high affinity to the intron RNA, occluding the Shine-Dalgarno sequence necessary for translation of LtrA, and presumably limiting access of the ribosome to structured regions of the group II ribozyme.

Structured group I introns interrupt the nuclear rDNA of many eukaryotes, in which coupled transcription and translation is not an issue, but they nonetheless face a different set of problems connected with intron-encoded ORFs. The well-studied group I introns in rDNA genes in the slime mold Didymium [62] contain ORFs known as IDirI and I-DirII. On transcription of the rDNA by polymerase I (Pol I), these ORFs are embedded within a transcript that is not able to be translated. How then can these proteins get expressed? In the case of I-DirII, the ORF is in the antisense orientation relative to the rDNA transcription unit, and expression of I-DirII is driven by its own RNA polymerase II promoter, followed by removal of a spliceosomal intron and addition of a poly(A) tail [63]. I-DirI is in the same orientation as the Pol I rRNA transcript [64], and has a more complicated expression mechanism. Maturation of a transcript competent for translation involves excision of an unusual branching ribozyme (known as DiGIR1) from the 5' end of the intron that generates a 2'-5' cap structure [65]. This is followed by processing of the 3' end and addition of a poly(A) tail. These types of regulation imply an extraordinary degree of co-evolution between intron, IEP and host gene that can best be explained by selective pressures to regulate intron splicing and ORF expression so as to not impart any phenotypic cost associated with expression of the (often essential) interrupted host gene. 
As noted earlier, many proteins encoded within introns in organellar genomes are initially translated as fusions with upstream exon sequences, requiring subsequent proteolytic processing to provide an active protein with an amino terminus in domain IV $[40,41,66]$. Little is known about the molecular machinery required for this process, due in part to the technically demanding nature of organellar biology, but this arrangement creates opportunities for regulatory cross-talk between translation of the upstream exon and splicing, in ways that need to be determined experimentally.

\section{Host factors that regulate mobility: mutualism or repression?}

Host-encoded proteins function to stimulate the splicing of group I and II introns. In the case of group II introns, host-function-assisted splicing is also crucial for mobility, as the spliced intron RNA is an active intermediate in the mobility pathway [67]. Detailed biochemical and structural studies have shown that host proteins function as maturases to stabilize the active group I or II RNA structure, as chaperones to resolve 'kinetic traps' that limit the rate of RNA folding, or as transporters to ensure the level of $\mathrm{Mg}^{2+}$ is sufficient for efficient folding and splicing. The requirement for host-encoded proteins is especially evident for many organellar group II introns: at least 14 nuclear gene products promote efficient splicing of the two group II introns in the chloroplast-encoded psaA gene of Chlamydomonas reinhardtii [68,69]. Another example of host-facilitated intron splicing involves the $\mathrm{Mg}^{2+}$ transporter Mrs2p, and the chaperone activity of three DEAD-box proteins, Mss116p, Ded1p and Cyt19p, to promote group II intron splicing in the mitochondria of fungi [70-74].

In terms of mobility, the primary response of a host genome to the presence of mobile elements is repressive, as unregulated mobile element activity will lead to an unbearable mutational load. In recent years, a number of studies have uncovered host proteins that downregulate the activities of mobile introns, many of which (not unexpectedly) are involved in aspects of RNA processing. These proteins include RNase E and RNase I, which negatively regulate group II intron mobility by reducing the steady-state level of intron RNA [75-77]. At the same time, a greater appreciation of the intricate relationships between introns and host factors that stimulate their mobility has arisen from observations that group I and II introns are obligately dependent on host-encoded functions to complete the mobility process. In the case of group I introns, the involvement of the intron-encoded homing endonuclease in mobility is limited to the introduction of a DSB (or of a single-strand nick [78,79], depending on the endonuclease) in cognate alleles that lack the intron. Completion of the mobility process requires host-encoded proteins that function in DNA recombination, replication and repair pathways [80-82]. Likewise, the retromobility pathways of group II introns are dependent on host machinery, as illustrated by the Ll.LtrB intron in Escherichia coli where host factors, including the major replicative polymerase Pol III, repair polymerases Pol II, Pol IV and Pol V, the endonuclease RNase $\mathrm{H} 1$, and DNA ligase, all function to complete a retromobility event [75]. Thus, the two intron types exploit different aspects of the host's nucleic acid transaction pathways.

\section{Molecular lifeboats - abandon ship!}

Up to this point we have considered the dynamic interplay between introns, their intramolecular inhabitants and their hosts, without considering evolutionary and environmental factors that might influence these partnerships. One traditional view of introns is that they are purely selfish DNA elements, imparting neither benefit nor burden to the host genome in which they reside. Recent evidence, however, has forced a re-evaluation of this viewpoint, particularly in the light of experimental data showing that introns can mobilize in response to stress-induced conditions [77,83], as has been demonstrated for other mobile elements $[84,85]$. These data raise the fascinating possibility that introns are 'plugged' into host metabolic pathways in ways that control and favor intron dissemination in times of environmental stress (Figure 3).

For instance, the group I intron endonuclease I-TevI (described in Figure 2) is subject to post-translational control under oxidative stress [83]. A zinc finger in an interdomain linker of I-TevI is redox-sensitive, and under oxidizing conditions is disrupted by loss of the zinc ion, leading to spurious DNA cleavage and intron movement to sites less similar in sequence to its usual allelic target. Reducing conditions restore zinc-finger function, cleavage and homing fidelity. This redox-responsive zincion cycling suggests a mechanism for rapid, regulated group I intron dispersal under conditions of oxidative stress (Figure 3).

Group II introns respond to metabolic stress with a burst of retrotransposition to new sites by a mechanism different from that used by group I introns. Retrotransposition of the lactococcal Ll.LtrB group II intron in E. coli is not only regulated by RNase E [76], but is also wired into the cell's global genetic circuitry via the two small-molecule effectors ppGpp and cAMP [77]. These global regulators, which are elevated during the 'stringent response' to amino acid starvation and upon glucose starvation, respectively, stimulate retrotransposition. Whereas the RNase E effect is mediated at the level of the invading intron RNA, the global regulators are proposed to act by stalling of chromosomal replication forks and/ 


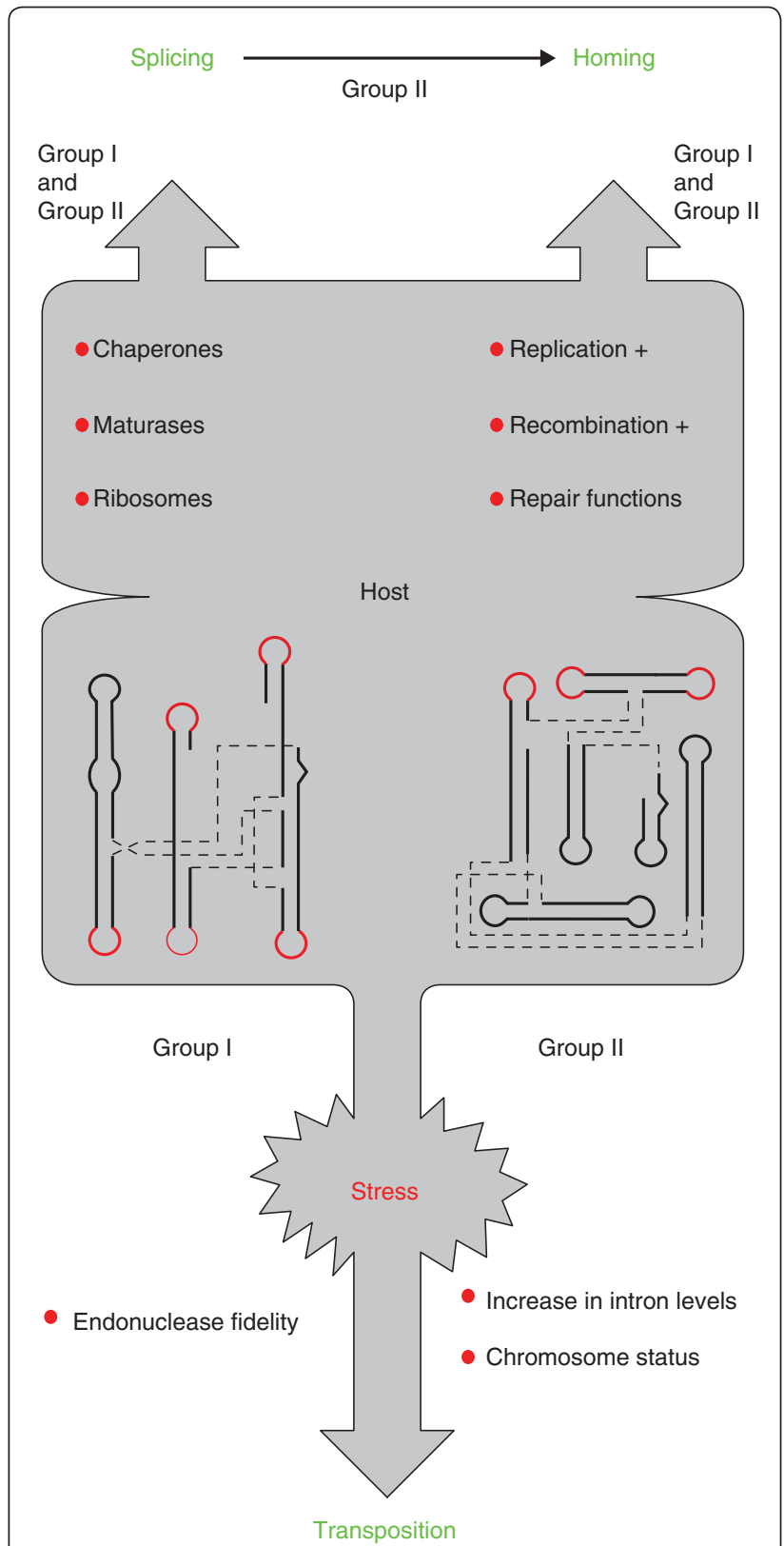

Figure 3. Model for intron-host interactions. The top half of the figure indicates that RNA chaperones and, sometimes, maturases and/or ribosomes are required to facilitate splicing of group I and group II introns, whereas replication, recombination and repair functions are necesary for homing of these elements in a host cell in a well balanced growth environment $[80,95]$. Splicing of group II introns is, in turn, required for their mobility [15]. The bottom of the figure indicates how mobile introns respond to stress conditions in their host cell. For group I introns, oxidative stress results in group I endonuclease substrate infidelity, allowing transposition of the intron to sites with less sequence similarity than the normal allelic target [83]. For group II introns, nutritional stress increases their rate of transcription, thus raising the level of intron RNA, and also alters the disposition of the nucleoid (the bacterial DNA) in ways that

favor retrotransposition; together these changes result in a burst of retrotransposition of group II introns in response to the stress [77]. or altering the transcriptional state of the nucleoid (that is, chromosome status), both of which might provide introns with access to the genome (Figure 3).

Clearly, the mechanisms whereby these variant introns respond to oxidative and nutritional stresses in order to disseminate are different, but with similar outcomes the 'abandoning of ship' for more hospitable genomic environs. For the group I intron, the mobility machinery itself, the intron endonuclease I-TevI, transduces the signal [83]. Whereas intron levels can also affect retrotransposition of the group II intron [76], the signal can, in addition, be transmitted through changes in macromolecular disposition of the host [77]. One (yet to be demonstrated) evolutionary consequence of this coupling between sensing of environmental conditions and intron dissemination is the potential to generate genetic novelties that are useful to the cell under stress. A documented mechanism for introns to generate genetic diversity is through alternative splicing pathways [86,87]. In bacteriophage Twort, which infects Staphylococcus aureus, the ORF orf182 is interrupted by three similar group I introns, and analysis of spliced products revealed that some transcripts lack one exon, suggestive of programmed exon skipping [88]. Similarly, trans-splicing between highly similar group II introns in organellar genomes also has the potential to generate novel transcripts [89]. It is tempting to speculate that these alternative splicing events can be regulated by the host to generate novel protein products under specific cellular conditions.

\section{Evolving perceptions about self-splicing introns}

Recent results have challenged our perceptions regarding self-splicing introns, from the notion that they represent simple genomic parasites imparting neither cost nor benefit to the host genome, to that of sophisticated mobile DNA elements fully integrated into host-cell metabolism in ways that could be viewed as molecular mutualism. Host organisms devote considerable resources, whether by design or accident, to both positively and negatively influence intron behavior, and elucidating the molecular basis of host-factor involvement in the regulation of intron splicing and mobility is one area ripe for future investigation. In particular, the mechanism underlying the processing of intron ORFs that are initially translated as fusion proteins with upstream exons in organellar introns represents an obvious gap in our knowledge, but this is a technically daunting problem to address. However, it is questions of an evolutionary slant that will challenge intronologists for years to come. Foremost among these is the possibility that introns could provide some benefit to hosts by generating genetic diversity as a consequence of transposition events brought on by cellular stress. 


\section{Acknowledgements}

We thank John Dansereau for assistance with figures, and Anna Pyle and Steve Zimmerly for discussion. This work was supported by a CIHR Operating Grant (MOP-97780) to DRE, a CIHR New Investigator Salary Award to DRE, and by NIH Grants GM39422 and GM44844 to MB.

\section{Author details}

'Department of Biochemistry, Schulich School of Medicine and Dentistry, The University of Western Ontario, London, Ontario, Canada N6A 5C1. ${ }^{2}$ Center for Medical Sciences, Wadsworth Center, New York State Department of Health, and University at Albany, SUNY, Albany, NY 12208, USA. ${ }^{3}$ National Cancer Institute, National Institutes of Health, Bethesda, MD 20892, USA

\section{References}

1. Doolittle WF, Sapienza C: Selfish genes, the phenotype paradigm and genome evolution. Nature 1980, 284:601-603.

2. Orgel LE, Crick FH: Selfish DNA: the ultimate parasite. Nature 1980, 284:604-607.

3. Sijen T, Plasterk RH: Transposon silencing in the Caenorhabditis elegans germ line by natural RNAi. Nature 2003, 426:310-314

4. Vagin W, Sigova A, Li C, Seitz H, Gvozdev V, Zamore PD: A distinct small RNA pathway silences selfish genetic elements in the germline. Science 2006, 313:320-324.

5. Kato M, Miura A, Bender J, Jacobsen SE, Kakutani T: Role of CG and non-CG methylation in immobilization of transposons in Arabidopsis. Curr Biol 2003, 13:421-426.

6. Jiang YW: Transcriptional cosuppression of yeast Ty1 retrotransposons. Genes Dev 2002, 16:467-478.

7. Kleckner N: Regulation of transposition in bacteria. Annu Rev Cell Bio/ 1990 6:297-327.

8. Cambareri EB, Foss HM, Rountree MR, Selker EU, Kinsey JA: Epigenetic control of a transposon-inactivated gene in Neurospora is dependent on DNA methylation. Genetics 1996, 143:137-146.

9. Aravin AA, Hannon GJ, Brennecke J: The Piwi-piRNA pathway provides an adaptive defense in the transposon arms race. Science 2007, 318:761-764.

10. Belfort M: Bacteriophage introns: parasites within parasites? Trends Genet 1989, 5:209-213.

11. Belfort M, Derbyshire V, Cousineau B, Lambowitz A: Mobile introns: pathways and proteins. In Mobile DNA II. Edited by Craig N, Craigie R, Gellert M, Lambowitz A. New York: ASM Press; 2002:761-783

12. Haugen $P$, Simon DM, Bhattacharya D: The natural history of group I introns. Trends Genet 2005, 21:111-119.

13. Cech TR, Damberger SH, Gutell RR: Representation of the secondary and tertiary structure of group I introns. Nat Struct Bio/ 1994, 1:273-280.

14. Lambowitz AM, Zimmerly S: Mobile group II introns. Annu Rev Genet 2004, 38:1-35.

15. Lambowitz AM, Perlman PS: Involvement of aminoacyl-tRNA synthetases and other proteins in group I and group II intron splicing. Trends Biochem Sci 1990, 15:440-444

16. Paukstelis PJ, Chen JH, Chase E, Lambowitz AM, Golden BL: Structure of a tyrosyl-tRNA synthetase splicing factor bound to a group I intron RNA. Nature 2008, 451:94-97.

17. Labouesse M, Netter P, Schroeder R: Molecular basis of the 'box effect', a maturase deficiency leading to the absence of splicing of two introns located in two split genes of yeast mitochondrial DNA. Eur J Biochem 1984, 144:85-93.

18. Meng Q, Wang Y, Liu XQ: An intron-encoded protein assists RNA splicing of multiple similar introns of different bacterial genes. J Bio/ Chem 2005, 280:35085-35088

19. BolduC JM, Spiegel PC, Chatterjee P, Brady KL, Downing ME, Caprara MG, Waring RB, Stoddard BL: Structural and biochemical analyses of DNA and RNA binding by a bifunctional homing endonuclease and group I intron splicing factor. Genes Dev 2003, 17:2875-2888.

20. Belfort M: Two for the price of one: a bifunctional intron-encoded DNA endonuclease-RNA maturase. Genes Dev 2003, 17:2860-2863.

21. Bullerwell CE, Burger $G$, Lang BF: A novel motif for identifying rps3 homologs in fungal mitochondrial genomes. Trends Biochem Sci 2000, 25:363-365.

22. Burke JM, RajBhandary UL: Intron within the large rRNA gene of $N$. crassa mitochondria: a long open reading frame and a consensus sequence possibly important in splicing. Cell 1982, 31:509-520.

23. Medina M, Collins AG, Takaoka TL, Kuehl JV, Boore JL: Naked corals: skeleton loss in Scleractinia. Proc Natl Acad Sci U S A 2006, 103:9096-9100.
24. Nielsen H, Johansen SD: Group I introns: Moving in new directions. RNA Biol 2009, 6:375-383

25. Braun V, Mehlig M, Moos M, Rupnik M, Kalt B, Mahony DE, von Eichel-Streiber C: A chimeric ribozyme in clostridium difficile combines features of group I introns and insertion elements. Mol Microbio/ 2000, 36:1447-1459.

26. Lee ER, Baker JL, Weinberg Z, Sudarsan N, Breaker RR: An allosteric selfsplicing ribozyme triggered by a bacterial second messenger. Science 2010, 329:845-848

27. Shub DA, Gott JM, Xu MQ, Lang BF, Michel F, Tomaschewski J, Pedersen-Lane J, Belfort M: Structural conservation among three homologous introns of bacteriophage T4 and the group I introns of eukaryotes. Proc Natl Acad SCi USA 1988, 85:1151-1155.

28. Shub DA, Goodrich-Blair H, Eddy SR: Amino acid sequence motif of group intron endonucleases is conserved in open reading frames of group II introns. Trends Biochem Sci 1994, 19:402-404.

29. Pyle AM: The tertiary structure of group II introns: implications for biological function and evolution. Crit Rev Biochem Mol Biol 2010, 45:215-232.

30. Michel F, Ferat JL: Structure and activities of group II introns. Annu Rev Biochem 1995, 64:435-461.

31. Toor N, Zimmerly S: Identification of a family of group II introns encoding LAGLIDADG ORFs typical of group I introns. RNA 2002, 8:1373-1377.

32. Mullineux ST, Costa M, Bassi GS, Michel F, Hausner G: A group II intron encodes a functional LAGLIDADG homing endonuclease and self-splices under moderate temperature and ionic conditions. RNA 2010, 16:1818-1831.

33. Copertino DW, Hallick RB: Group II twintron: an intron within an intron in a chloroplast cytochrome b-559 gene. EMBO J 1991, 10:433-442.

34. Nakamura Y, Kaneko T, Sato S, Ikeuchi M, Katoh H, Sasamoto S, Watanabe A, Iriguchi M, Kawashima K, Kimura T, Kishida Y, Kiyokawa C, Kohara M, Matsumoto M, Matsuno A, Nakazaki N, Shimpo S, Sugimoto M, Takeuchi C, Yamada M, Tabata S: Complete genome structure of the thermophilic cyanobacterium Thermosynechococcus elongatus BP-1. DNA Res 2002, 9:123-130

35. Mohr G, Ghanem E, Lambowitz AM: Mechanisms used for genomic proliferation by thermophilic group II introns. PLOS Bio/ 2010, 8:e1000391.

36. Glanz S, Kuck U: Trans-splicing of organelle introns - a detour to continuous RNAs. Bioessays 2009, 31:921-934.

37. Toor N, Keating KS, Taylor SD, Pyle AM: Crystal structure of a self-spliced group II intron. Science 2008, 320:77-82.

38. Simon DM, Clarke NA, McNeil BA, Johnson I, Pantuso D, Dai L, Chai D, Zimmerly S: Group II introns in eubacteria and archaea: ORF-less introns and new varieties. RNA 2008, 14:1704-1713.

39. Zhou L, Manias DA, Dunny GM: Regulation of intron function: efficient splicing in vivo of a bacterial group II intron requires a functional promoter within the intron. Mol Microbiol 2000, 37:639-651.

40. Carignani G, Groudinsky O, Frezza D, Schiavon E, Bergantino E, Slonimski PP: An mRNA maturase is encoded by the first intron of the mitochondrial gene for the subunit I of cytochrome oxidase in S. cerevisiae. Cell 1983, 35:733-742.

41. Moran JV, Mecklenburg KL, Sass P, Belcher SM, Mahnke D, Lewin A, Perlman P: Splicing defective mutants of the COXI gene of yeast mitochondrial DNA: initial definition of the maturase domain of the group II intron al2. Nucleic Acids Res 1994, 22:2057-2064.

42. Bacher G, Szymanski WW, Kaufman SL, Zollner P, Blaas D, Allmaier G: Chargereduced nano electrospray ionization combined with differential mobility analysis of peptides, proteins, glycoproteins, noncovalent protein complexes and viruses. J Mass Spectrom 2001, 36:1038-1052.

43. Bonocora RP, Shub DA: A likely pathway for formation of mobile group I introns. Curr Bio/ 2009, 19:223-228.

44. Loizos N, Tillier ER, Belfort M: Evolution of mobile group I introns: recognition of intron sequences by an intron-encoded endonuclease. Proc Natl Acad Sci USA 1994, 91:11983-11987.

45. Toor N, Hausner G, Zimmerly S: Coevolution of group II intron RNA structures with their intron-encoded reverse transcriptases. RNA 2001, 7:1142-1152

46. Robart AR, Zimmerly S: Group II intron retroelements: function and diversity. Cytogenet Genome Res 2005, 110:589-597.

47. Van Roey P, Waddling CA, Fox KM, Belfort M, Derbyshire V: Intertwined structure of the DNA-binding domain of intron endonuclease I-Tevl with its substrate. EMBO J 2001, 20:3631-3637.

48. Yang J, Zimmerly S, Perlman PS, Lambowitz AM: Efficient integration of an intron RNA into double-stranded DNA by reverse splicing. Nature 1996, 381:332-335 
49. Ohman-Heden M, Ahgren-Stalhandske A, Hahne S, Sjoberg BM: Translation across the $5^{\prime}$-splice site interferes with autocatalytic splicing. Mol Microbiol 1993, 7:975-982

50. Sandegren L, Sjoberg BM: Self-splicing of the bacteriophage T4 group I introns requires efficient translation of the pre-mRNA in vivo and correlates with the growth state of the infected bacterium. J Bacterio/ 2007, 189:980-990

51. Semrad K, Schroeder R: A ribosomal function is necessary for efficient splicing of the T4 phage thymidylate synthase intron in vivo. Genes Dev 1998, 12:1327-1337.

52. Bonocora RP, Shub DA: A novel group I intron-encoded endonuclease specific for the anticodon region of tRNA(fMet) genes. Mol Microbio/ 2001, 39:1299-1306

53. Bechhofer DH, Hue KK, Shub DA: An intron in the thymidylate synthase gene of Bacillus bacteriophage beta 22: evidence for independent evolution of a gene, its group I intron, and the intron open reading frame. Proc Natl Acad SciU SA 1994, 91:11669-11673.

54. Bonocora RP, Shub DA: A self-splicing group I intron in DNA polymerase genes of T7-like bacteriophages. J Bacterio/ 2004, 186:8153-8155.

55. Gott JM, Zeeh A, Bell-Pedersen D, Ehrenman K, Belfort M, Shub DA: Genes within genes: independent expression of phage T4 intron open reading frames and the genes in which they reside. Genes Dev 1988, 2:1791-1799.

56. Brok-Volchanskaya VS, Kadyrov FA, Sivogrivov DE, Kolosov PM, Sokolov AS, Shlyapnikov MG, Kryukov VM, Granovsky IE: Phage T4 SegB protein is a homing endonuclease required for the preferred inheritance of T4 tRNA gene region occurring in co-infection with a related phage. Nucleic Acids Res 2008, 36:2094-2105.

57. Gibb EA, Edgell DR: An RNA hairpin sequesters the ribosome binding site of homing endonuclease mobE gene. J Bacterio/ 2009, 191:2409-2413.

58. Petrov VM, Ratnayaka S, Karam JD: Genetic insertions and diversification of the PolB-type DNA polymerase (gp43) of T4-related phages. J Mol Biol 2010, 395:457-474

59. Gibb EA, Edgell DR: Better late than early: delayed translation of intronencoded endonuclease I-Tevl is required for efficient splicing of its host group I intron. Mol Microbiol 2010, 78:35-46.

60. Singh RN, Saldanha RJ, D'Souza LM, Lambowitz AM: Binding of a group II intron-encoded reverse transcriptase/maturase to its high affinity intron RNA binding site involves sequence-specific recognition and autoregulates translation. J Mol Biol 2002, 318:287-303.

61. Wank H, SanFilippo J, Singh RN, Matsuura M, Lambowitz AM: A reverse transcriptase/maturase promotes splicing by binding at its own coding segment in a group II intron RNA. Mol Cell 1999, 4:239-250.

62. Johansen S, Einvik C, Nielsen H: DiGIR1 and NaGIR1: naturally occurring group I-like ribozymes with unique core organization and evolved biological role. Biochimie 2002, 84:905-912.

63. Johansen SD, Vader A, Sjottem E, Nielsen H: In vivo expression of a group I intron HEG from the antisense strand of Didymium ribosomal DNA. RNA Biol 2006, 3:157-162.

64. Vader A, Johansen S, Nielsen H: The group I-like ribozyme DiGIR1 mediates alternative processing of pre-rRNA transcripts in Didymium iridis. Eur J Biochem 2002, 269:5804-5812.

65. Nielsen $\mathrm{H}$, Westhof $\mathrm{E}$, Johansen $\mathrm{S}$ : An mRNA is capped by a 2', 5' lariat catalyzed by a group I-like ribozyme. Science 2005, 309:1584-1587.

66. Guo WW, Moran JV, Hoffman PW, Henke RM, Butow RA, Perlman PS: The mobile group I intron 3 alpha of the yeast mitochondrial COXI gene encodes a 35-kDa processed protein that is an endonuclease but not a maturase. J Biol Chem 1995, 270:15563-15570.

67. Zimmerly S, Guo H, Eskes R, Yang J, Perlman PS, Lambowitz AM: A group II intron RNA is a catalytic component of a DNA endonuclease involved in intron mobility. Cell 1995, 83:529-538.

68. Goldschmidt-Clermont M, Girard-Bascou J, Choquet Y, Rochaix JD: Transsplicing mutants of Chlamydomonas reinhardtii. Mol Gen Genet 1990, 223:417-425.

69. Rivier C, Goldschmidt-Clermont M, Rochaix JD: Identification of an RNAprotein complex involved in chloroplast group II intron trans-splicing in Chlamydomonas reinhardtii. EMBO J 2001, 20:1765-1773.

70. Del Campo M, Mohr S, Jiang Y, Jia H, Jankowsky E, Lambowitz AM: Unwinding by local strand separation is critical for the function of DEADbox proteins as RNA chaperones. J Mol Biol 2009, 389:674-693.

71. Solem A, Zingler N, Pyle AM: A DEAD protein that activates intron selfsplicing without unwinding RNA. Mol Cell 2006, 24:611-617.
72. Fedorova O, Solem A, Pyle AM: Protein-facilitated folding of group II intron ribozymes. J Mol Biol 2010, 397:799-813.

73. Huang HR, Rowe CE, Mohr S, Jiang Y, Lambowitz AM, Perlman PS: The splicing of yeast mitochondrial group I and group II introns requires a DEAD-box protein with RNA chaperone function. Proc Natl Acad Sci U SA 2005, 102:163-168.

74. Halls C, Mohr S, Del Campo M, Yang Q, Jankowsky E, Lambowitz AM: Involvement of DEAD-box proteins in group I and group II intron splicing. Biochemical characterization of Mss116p, ATP hydrolysis-dependent and -independent mechanisms, and general RNA chaperone activity. J Mol Biol 2007, 365:835-855

75. Smith D, Zhong J, Matsuura M, Lambowitz AM, Belfort M: Recruitment of host functions suggests a repair pathway for late steps in group II intron retrohoming. Genes Dev 2005, 19:2477-2487.

76. Coros CJ, Piazza CL, Chalamcharla VR, Belfort M: A mutant screen reveals RNase $\mathrm{E}$ as a silencer of group II intron retromobility in Escherichia coli. RNA 2008, 14:2634-2644.

77. Coros CJ, Piazza CL, Chalamcharla VR, Smith D, Belfort M: Global regulators orchestrate group II intron retromobility. Mol Cell 2009, 34:250-256.

78. Landthaler M, Lau NC, Shub DA: Group I intron homing in Bacillus phages SPO1 and SP82: a gene conversion event initiated by a nicking homing endonuclease. J Bacterio/ 2004, 186:4307-4314

79. Wilson GW, Edgell DR: Phage T4 mobE promotes trans homing of the defunct homing endonuclease I-TevllI. Nucleic Acids Res 2009, 37:7110-7123.

80. Mueller JE, Clyman J, Huang YJ, Parker MM, Belfort M: Intron mobility in phage T4 occurs in the context of recombination-dependent DNA replication by way of multiple pathways. Genes Dev 1996, 10:351-364.

81. Parker MM, Belisle M, Belfort M: Intron homing with limited exon homology. Illegitimate double-strand-break repair in intron acquisition by phage T4. Genetics 1999, 153:1513-1523

82. Huang YJ, Parker MM, Belfort M: Role of exonucleolytic degradation in group I intron homing in phage T4. Genetics 1999, 153:1501-1512.

83. Robbins JB, Smith D, Belfort M: Redox-responsive zinc finger fidelity switch in homing endonuclease and intron promiscuity in oxidative stress. Curr Biol 2011, 21:243-248

84. Lesage P, Todeschini AL: Happy together: the life and times of Ty retrotransposons and their hosts. Cytogenet Genome Res 2005, 110:70-90.

85. Wardle SJ, O'Carroll M, Derbyshire KM, Haniford DB: The global regulator $\mathrm{H}-\mathrm{NS}$ acts directly on the transpososome to promote $\mathrm{Tn} 10$ transposition. Genes Dev 2005, 19:2224-2235

86. Sellem CH, Belcour L: The in vivo use of alternate 3 '-splice sites in group I introns. Nucleic Acids Res 1994, 22:1135-1137.

87. Sellem $\mathrm{CH}$, Belcour $\mathrm{L}$ : Intron open reading frames as mobile elements and evolution of a group I intron. Mol Biol Evol 1997, 14:518-526.

88. Landthaler M, Shub DA: Unexpected abundance of self-splicing introns in the genome of bacteriophage Twort: introns in multiple genes, a single gene with three introns, and exon skipping by group I ribozymes. Proc Natl Acad Sci U S A 1999, 96:7005-7010.

89. Hiller R, Hetzer M, Schweyen RJ, Mueller MW: Transposition and exon shuffling by group II intron RNA molecules in pieces. J Mol Biol 2000, 297:301-308.

90. Nord D, Torrents E, Sjöberg B-M: A functional homing endonuclease in the Bacillus anthracis nrdE group I intron. J Bacterio/ 2007, 189:5293-5301.

91. Goodrich-Blair H, Shub DA: The DNA polymerase genes of several HMUbacteriophages have similar group I introns with highly divergent open reading frames. Nucleic Acids Res 1994, 22:3715-3721.

92. Haugen P, Bhattacharya D, Palmer JD, Turner S, Lewis LA, Pryer KM: Cyanobacterial ribosomal RNA genes with multiple, endonucleaseencoding group I introns. BMC Evol Bio/ 2007, 7:159.

93. Biniszkiewicz D, Cesnaviciene E, Shub DA: Self-splicing group I intron in cyanobacterial initiator methionine tRNA: evidence for lateral transfer of introns in bacteria. EMBO J 1994, 13:4629-4635

94. Muscarella DE, Vogt VM: A mobile group I intron in the nuclear rDNA of Physarum polycephalum. Cell 1989, 56:443-454.

95. Beauregard A, Curcio MJ, Belfort M: The take and give between retrotransposable elements and their hosts. Annu Rev Genet 2008, 42:587-617.

doi:10.1186/1741-7007-9-22

Cite this article as: Edgell DR, et al.: Learning to live together: mutualism between self-splicing introns and their hosts. BMC Biology 2011, 9:22. 